tension which can be relieved fully by eserin, should ordinarily be operated upon without preliminary miotic treatment lasting for a period proportionate to the degree and probable duration of the tension. A period of two to three months would be the maximum, as far as it can be judged from the recurrences after operation.

\title{
Selection of Operation
}

The above division of chronically glaucomatous eyes into two groups, according to completeness or incompleteness of response to miotics, may be of service in indicating the type of operation suitable to individual cases.

The one group may possibly contain all the cases in which such a simple measure as flap sclerotomy may be expected to succeed. Does it include only these ? Pending further development of my thread sclerotomy (Trans. Ophthal. Soc. U. K., 1919), should not some very advanced mild glaucomas, with damaged central vision, be subjected rather to the wide prolapse treatment, even though the tension may give way quite readily to eserin?

The prolapse operation may be perhaps at present the soundest treatment for all primary glaucomas not fully reducible by eserin, and not already operated upon by wide iridectomy. Certainly, the application of the method promises to be much wider than was suggested in the Transactions (1919), p. 218.

For use in India I think it is the best routine general operation, suited to the capacity of the average civil surgeon.

The existence of a wide coloboma, while barring the prolapse operation, renders an eye particularly suited to thread sclerotomy. In this operation the only real difficulty lies in preventing adhesion of iris to the wound.

These three operations cover nearly the whole field of primary glaucoma, combining safety with efficiency. Together they go far towards the final solution of the main glaucoma question, which has not proved to be so simple as it once seemed to be.

THE TREATMENT OF PROLAPSE OF THE IRIS FOLLOWING ACCIDENTAL PERFORATING WOUNDS, WITH A NOTE ON THE REMOVAL OF NON-MAGNETIC FOREIGN BODIES FROM THE ANTERIOR CHAMBER * BY

Charles Goulden, O.B.E., M.D., M.C., F.R.C.S.

PROLAPSE of the iris in connection with accidental wounds of the eye is a fairly frequent occurrence. Many cases are not difficult to

\footnotetext{
* Read in the Section of Ophthalmology of the Royal Society of Medicine on March 3rd, 1920.
} 
deal with, but on the other hand there are cases, especially when the wound in the cornea or corneo-sclera is an extensive or irregular one, which are difficult to manage.

Individual experience, even over a long period, is never very extensive, but the numerous eye injuries that occurred during the war gave to those who were in charge of ophthalmic wards over-seas an extraordinary opportunity of dealing with, not only great numbers, but an infinite variety of wounds of the eye associated with prolapse of the uvea.

Attempts at reposition of prolapsed iris should be avoided on account of the risk of infection of the interior of the eye, and because such a procedure is useless as the reposed iris will again prolapse.

Every attempt should be made, not only to remove the piece of iris prolapsed, but also to clear the wound of uvea; if left with iris incarcerated one or other of the following undesirable conditions will follow :

(a) Delayed healing of the wound;

(b) A weak scar which may bulge before the intraocular pressure ;

(c) Defective vision due to a displacement of the pupil and irregular refraction of the cornea ;

(d) Iridocyclitis ;

(e) Sympathetic iridocyclitis.

In any event, the appearance of the eye is much better with a well made iridectomy than with a distorted pupil and a leukoma adhaerens. When undertaking the removal of a prolapse of the iris a great deal depends upon the length of time that has passed since the accident and no doubt cases that come into the hands of the surgeon several days after the iris first prolapsed are very much more difficult to deal with. It is impossible to lay down a definite time after which it is impossible or not advisable to operate. Cases can certainly be operated upon and the wound freed of uvea as long as ten days after the accident. A method of operating which makes these cases more easy to deal with will be mentioned a little later on.

It will be convenient to divide the cases into groups as follow:

(1) Cases in which the wound is purely corneal,

(2) Cases in which the wound is at the corneo-scleral margin.

(3) Cases in which the wound extends past the limbus into the sclera.

If the prolapse of iris is complicated by the presence of a magnetic foreign body, this should receive attention and be removed from the eye through a separate incision before the prolapse is dealt with. 
As a rule the patient requires a general anaesthetic; this will allow deliberate operating and the surgeon will be able to pull the iris in any direction he may consider necessary without feeling anxious lest the patient make. some sudden movement which may lead to extensive tearing of the iris and bleeding that will hide everything that it is necessary to see so as to complete the operation properly.

1. Cases in which the wound is purely corneal.

If the wound be small these cases are the easiest to treat. The prolapsed knuckle of iris should be seized in toothed iris forceps, held in the right hand and the iris gently pulled in each direction,

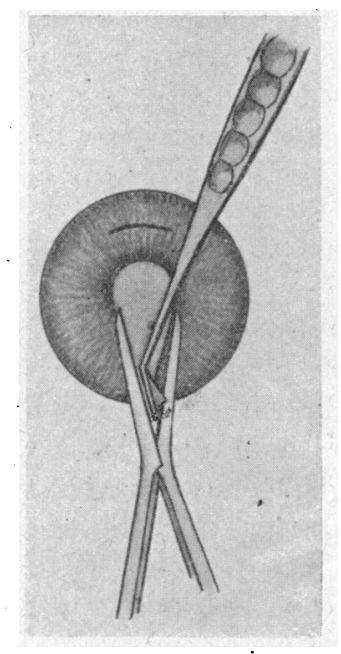

FIG. 1.

Showing the method of using Couper's capsule forceps in seizing the prolapse of iris along its whole length. In the upper part of the cornea is shown the incision recommended for the use of the repositor in difficult cases.

from side to side, to and from the ciliary attachment, the iris within the eye being observed all the time. It is important to make certain that there is perfect freedom of the iris where it is grasped by the edges of the corneal wound. Whilst drawing gently upon the iris seize, in the plane of the anterior surface of the cornea, the whole length of the prolapse with Couper's capsule forceps (Fig. 1), release the hold with the iris forceps, and drawing gently with the Couper's forceps, cut off the iris with de Wecker's scissors, pressing them gently upon the cornea whilst cutting. This cut must be done with one snip of the scissors, as the haemorrhage which follows most likely will obscure the contents of the anterior chamber and will not allow a second attempt to be made with safety to the lens capsule. Usually the iris springs back into the anterior chamber leaving the pillars of the consequent coloboma quite free. 
What has been done is, in effect, this : A portion of the iris not previously prolapsed has been drawn outside the eye, the iris has been put on the stretch, and the cut with the scissors has been made through healthy iris drawn outside the eye by the operator.

Now, wounds of the cornea are usually radial, the perforating instrument piercing the eye directly from before backwards: It is in such cases that treatment is not difficult, but when the wound of the cornea is ragged, flap-like, or tangential (like a wound made with a keratome), the problem of freeing the corneal wound completely is much more difficult. It is not difficult to free that portion of the iris that followed the path of withdrawal of the perforating instrument, as the repositor can be used by passing it into the eye in the same plane and direction in which the perforating instrument entered the eye, but the repositor, however bent or manipulated, cannot be made to engage that portion of the iris that lies under the lower lip of the valve-shaped wound, and consequently that pillar of the coloboma is often left still engaged in or adherent to the wound in the cornea.

The plan that has been used with success in such cases is as follows :

Before attempting to deal with the prolapse of iris, and whilst there is still an anterior chamber, make a small incision in the cornea at a point just opposite the accidental wound (Fig. 1), and three millimetres within the limbus; the wound need not be more than four millimetres long. Now deal with the prolapse and free it as well as possible. After this the repositor may be used by inserting it within the eye through the incision made with the keratome and the pillars of the coloboma replaced. If a foreign body has been removed previously, the incision made for its removal may be used for inserting the repositor.

The above remarks apply to a case in which operation is undertaken within twenty-four hours of the accident, and before any firm adhesions have formed between the prolapsed iris and the edges of the corneal wound. If more than a day has passed, and especially if several days have passed since the accident, the operation is much more difficult. The iris will be covered with greyish lymph and sodden with exudate where it is prolapsed and the edges of the corneal wound softened and grey with infiltration.

The lymph should be gently teased away from the iris with a repositor under a stream of warm saline solution, which will cause the prolapsed iris to be seen more easily, being washed here and there by the stream of lotion, for it must be remembered that the iris is often almost completely bleached and much attenuated. It is now seized and carefully freed. This is best done by paying attention to each part of the corneal wound in turn. Draw the prolapse away from one side, almost in the plane of the cornea, and 
whilst doing this gently insert the iris repositor between the lip of the corneal wound and the iris, breaking down adhesions all the time until it is possible to pull uninjured iris from within the eye all along the edge of the wound in the cornea. This must be repeated in all directions and then the iris cut off as described above.

2. Cases in which the wound is at the corneo-scleral margin.

In these cases the wound is not infrequently punctiform and although the wound will allow the iris to prolapse, it is not sufficiently large to admit the iris repositor after the iris has been removed. Here again the advantage of the preliminary corneal incision will be seen.

3. Cases in which the wound extends past the limbus, thus involving both cornea and sclera.

Here the corneo-scleral wound and subsequent prolapse of iris is complicated by a wound in the conjunctiva. As the conjunctiva, lacerated or cut, falls together and soon becomes adherent by its cut edges, it is difficult to judge the extent of the wound and the amount of uvea prolapsed: hence the importance of exposing freely the corneo-scleral wound and the inadvisability of attempting an operation until the conjunctiva has been drawn quite freely to one side.

With an ordinary strabismus scissors extend the conjunctival wound, undermining if necessary, and then having passed a suture through each edge, let your assistant draw the edges apart or attach a small artery forceps to the end of each suture and allow them to retract the wound by their own weight (Fig. 2). The corneo-

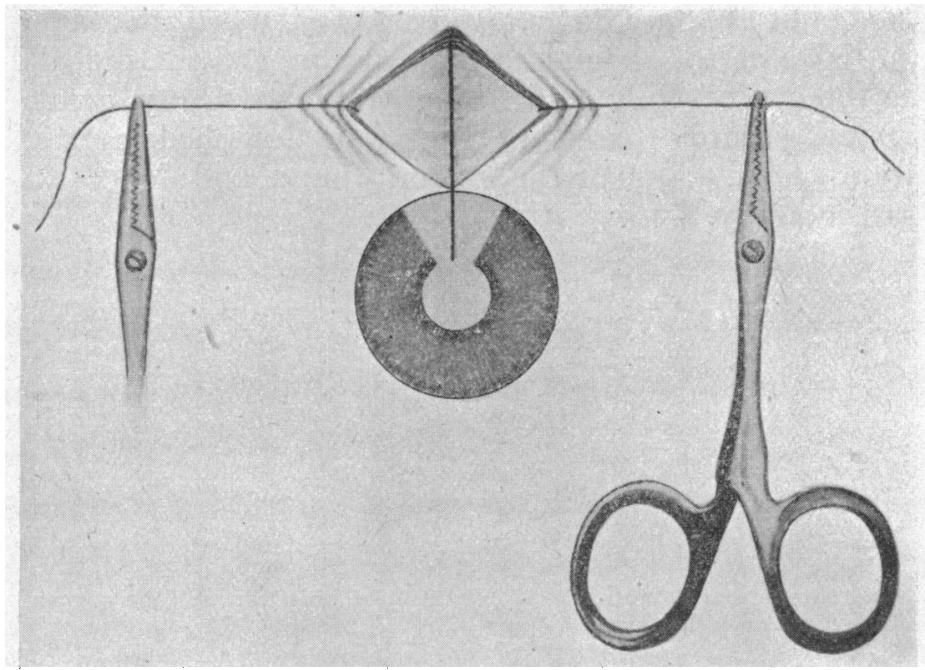

FIG. 2.

Showing the method of exposing a wound in the sclera by retracting the conjunctiva so as to deal with the uvea prolapsed. 
sclera is then beautifully. exposed and the prolapsed iris and ciliary body can be seen along their whole length and deliberately dealt with. It is important that such wound in the sclera should be completely covered with conjunctiva, and unless the edges of the conjunctiva fall together easily and without tension, the conjunctiva should be undermined around the scleral wound and detached from the limbus on each side by cutting round the edge of the cornea. To ensure immediate closure of the wound the conjunctiva should not be sutured edge to edge, but by two or more U-shaped sutures

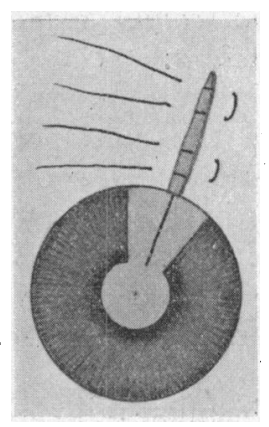

Fig. 3.

Showing the method of inserting sutures in the conjunctiva after dealing with such a case as that shown in Fig. 2.

(Fig. 3) passed a little distance from the edge of the conjunctival wound. The question of the use of scleral sutures will admit of discussion, but they are difficult to pass even when they are passed from the edge of the wound to the exterior, and the probability of bruising the eye severely and losing vitreous is very great. The conjunctival suture passed in the way described causes good adaptation of the edges of the scleral wound and braces them up quite satisfactorily.

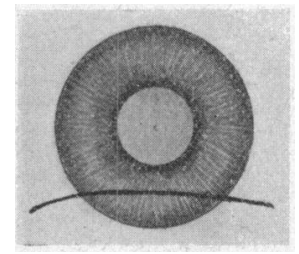

Fig. 4.

Showing an incised wound which extends not only through the cornea but also involves the sclera on either side.

A very troublesome type of case is one in which the wound has passed through the limbus on one side across the cornea and through the limbus on the other side (Fig. 4). These cases will 
only permit of conservative treatment when the wound is just within the limbus ; wounds which pass across the middle of the cornea will cause so much harm that the eye will have to be excised at once.

In those cases which allow an attempt to save the eye, wounds usually pass across the lower part of the cornea, and it will be found that after the prolapsed iris has been removed the edges of the corneal wound do not fall into apposition and that any chance of healing is very

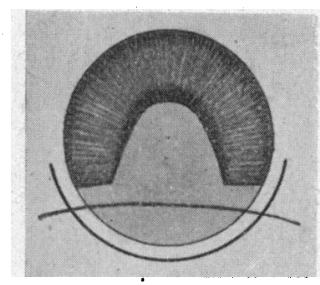

Fig. 5.

Showing the wound freed of uvea and the incision to be made round the cornea so as to detach the conjunctiva.

small and the risk of intraocular infection equally great. Much more is this the case when the prolapse has been present several days and the lower corneal flap accustomed, as it were, to its vicious position.

These wounds must therefore be liberally covered with conjunctiva, with the sutures so placed in the conjunctival as to help to approximate the edges of the corneal wound. The conjunctiva should be detached from the periphery of the cornea (Fig. 5) in

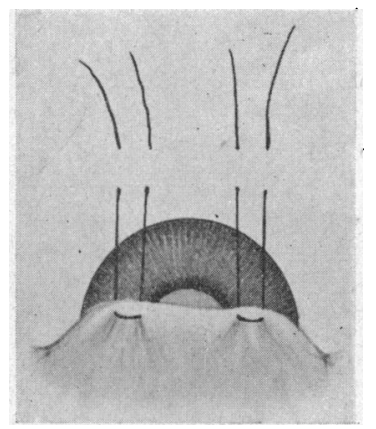

FIG. 6

Showing the manner of drawing the conjunctiva across to the cornea so as to bury a wound such as that shown in Fig. 5.

about its lower third or even one half and drawn straight across the cornea and sutured at the opposite limbus (Fig. 6). Such sutures will hold from five to seven days and when they have broken loose the conjunctiva will have become firmly adherent to the site of the injury. 


\section{A note on the removal of non-magnetic foreign bodies from the anterior chamber}

The removal of non-magnetic foreign bodies from the anterior chamber is usually not only very difficult but of extreme danger to the lens. It has been usual when a foreign body is lying on the iris (often in the lower part of the anterior chamber) to make an incision at the limbus with a keratome at the site of the foreign body (Fig. 7) and then introducing a curved iris forceps to try to seize the foreign body and so remove it. This often fails and then an iridectomy is made hoping to include the foreign body in the piece of iris removed.

The reason why it is so difficult or impossible to remove the foreign body in this way is largely due to the fact that the incision at the limbus is not on the same plane as the iris; and therefore not on the same plane as the foreign body; consequently it is not possible in so small a wound sufficiently to depress the points of the forceps used so as to hold the foreign body securely. If the foreign body be at the angle of the anterior chamber

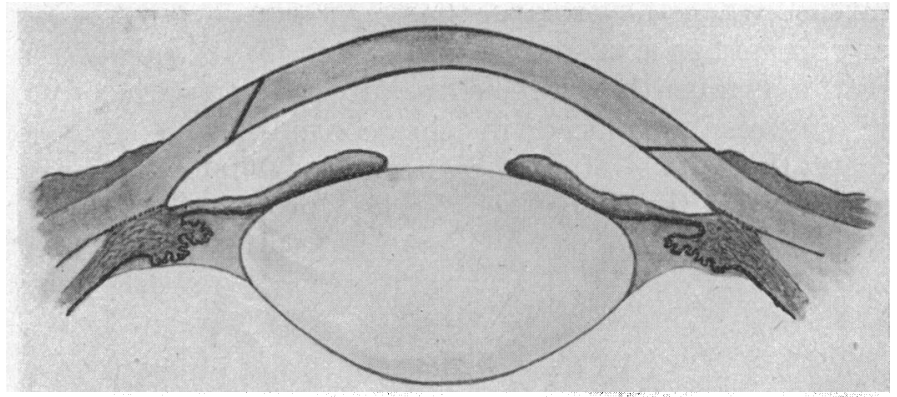

Fig. 7.

To show how it is that the usual incision at the limbus is unsuitable for the removal of a non-magnetic foreign body from the anterior chamber with forceps. If a foreign body be supposed to lie on the iris near the angle of the anterior chamber on the right side of the diagram then with an incision at the limbus the lower lip of the incision will not allow the foreign body to be seized.

If, however, a foreign body be supposed to lie on the iris near the angle of the anterior chamber on the left side of the diagram, it will be seen that the incision indicated over the foreign body affords a much easier approach and an easier field in which to use the forceps.

there is a lip of the sclera between the forceps and the foreign body round which it is not possible to manœuvre the point of the forceps (Fig. 7), and so the attempt must necessarily fail. Again, if the first attempt fails, the iris will in all probability be injured, and blood will cover the field of operation.

The following plan simplifies operation:

Make a keratome incision in the cornea halfway between the centre and the limbus (i.e., three millimetres inside the limbus) in 
the quadrant of the cornea lying over the foreign body (Fig. 7) and with the point of the keratome driven towards the foreign body. If the point of the keratome seems to drive too near the angle of the chamber the wound may be enlarged by side-toside movements instead of by an onward thrust. The foreign body may now be easily seized with toothless iris forceps and withdrawn. The risk of prolapse of iris is also avoided, as with corneal incisions in this situation the iris does not prolapse as is so often the case with incisions at the limbus.

In a case in which an eyelash was lying on the lens and no part lying on the iris a similar procedure was adopted, but the lash was first disengaged and gently coaxed over the iris by a stream of saline solution through the silver nozzle of a M'Keown's irrigator devised for the treatment of immature cataract.

\section{ON A SMALL IMPROVEMENT IN SKIN GRAFTING \\ BY}

\section{ERNEST E. MADDOX}

BOURNEMOUTH.

I find the cutting of Thiersch grafts to be facilitated by first painting the skin (after the usual preparation) with a stiffening agent, such as a thin even layer of flexible collodion.

This stiffens the epidermis, so that it moves over the knife with less crumpling, and it also makes the application of the graft much easier in certain difficult situations.

I would not, however, advise the use of a stiffening agent in all cases, since sometimes the very softness of the graft enables it the better to fill in the inequalities of an uneven surface.

Paraffin wax could be substituted for collodion when the latter is not obtainable, but although it would help the cutting, there is the danger of fragments becoming detached and getting in the way during the application of the graft. For this reason collodion or formalized gelatine is better.

\section{ARTIFICIAL EPISTAXIS \\ BY \\ ERnEst E. MADDOX \\ BOURNEMOUTH.}

A NOVEL, and I think valuable, expedient came to my rescue as a substitute for Heurtelouping when leeches became scarce during the war. In acute and sub-acute glaucoma and other congestions 